\title{
Eosinophilic granulomatous gastrointestinal and hepatic abscesses attributable to basidiobolomycosis and fasciolias: a simultaneous emergence in Iraqi Kurdistan
}

\author{
Hemmin A Hassan', Runnak A Majid', Nawshirwan G Rashid', Bryar E Nuradeen², Qalandar H Abdulkarim',
} Tahir A Hawramy ${ }^{2}$, Rekawt M Rashid ${ }^{3}$, Alton B Farris ${ }^{4}$, Jeannette Guarner ${ }^{4}$ and Michael D Hughson ${ }^{\text {** }}$

\begin{abstract}
Background: Deep eosinophilic granulomatous abscesses, as distinguished from eosinophilic subcutaneous abscesses, are rare. Most reports are from the Far-East and India where the most commonly attributed cause is Toxocara. Sulaimaniyah in Northeastern Iraq has experienced an outbreak of eosinophilic granulomatous liver and gastrointestinal (Gl) abscesses beginning in 2009. The purpose of this study was to determine the etiology and guide treatment.

Methods: The study was an ongoing investigation of patients having a histopathologic diagnosis of eosinophilic granulomatous abdominal abscesses in Sulaimaniyah hospitals from May 2009 to August 2012. Tissues were examined for organisms, and Enzyme Linked Immunoabsorbent Assays (ELISA) were performed for serum antibodies to Fasciola hepatica, Toxocara, and Echinococcus granulosus.
\end{abstract}

Results: Fourteen patients had granulomatous inflammation surrounding a central necrotizing eosinophilic exudate identified in surgical pathology specimens from abdominal surgeries. Two children and four adults had abscesses that formed Gl masses. These patients included a 39 year old male with oropharyngeal and transverse colon disease, and a 48 year old male with liver and Gl abscesses. All sites demonstrated a Zygomycete fungus surrounded by eosinophilic Splendori-Hoeppli material consistent with basidiobolomycosis. Five of the six patients with fungal disease were treated by surgery and 4 to 7 months of itraconozol. One child died of intestinal perforation while receiving IV amphotericin B; two adults required additional surgery for recurrent Gl obstruction. Eight patients had isolated liver abscesses with no organisms identified by histopathology: ELISA results for F. hepatica were positive for five, borderline for one, and negative for two patients. These eight patients were treated for fascioliasis by surgical resection of localized abscesses and albendazol. One patient serologically positive for F. Hepatica was found to have a common duct fluke two years after initial diagnosis. Serological testing for Toxocara and Echinococcus granulosus was negative in all 14 patients.

Conclusions: Basidiobolomycosis and F. hepatica are implicated as the cause of abdominal eosinophilic granulomatous abscesses in 12 of 14 patients identified over a period of 40 months in northern Iraq. Treatment was complicated by chronic biliary tract disease in fascioliasis and perforation and recurrent intestinal obstruction with basidiobolomycosis.

Keywords: Granulomas, Eosinophilia, Fascioliasis, Basidiobolomycosis, Gastrointestinal infections

\footnotetext{
* Correspondence: mhughson@bellsouth.net

'Departments of Pathology, Medicine, and Surgery, Shorsh General Hospital,

Sulaimaniyah, Iraq

Full list of author information is available at the end of the article
} 


\section{Background}

Eosinophilic and granulomatous liver and gastrointestinal (GI) abscesses, as distinguished from Langerhans cell disease, are barely mentioned in the pathology textbooks [1-6]. A few articles are available from the Far East, South America, and India where Toxocara as part of visceral larvae migrans is implicated, although often not proven, as being the etiologic agent [7-12]. In 2001, eosinophilic hepatic granulomas were reported in 43 cases from the files of the Armed Forces Institute of Pathology (AFIP) that were collected over 31 years [13]. Toxocara was identified in $30 \%$ of the specimens and was suggested to be the causative organism for the series. Based upon the experience with autopsies in Uruguay, Vercelli-Retta et al. [14,15] in a following letter to the editor cautioned that Fasciola hepatica may be involved in similar cases, if not actually some of those in the AFIP collection. The fungal disease basidiobolomycosis is an additional cause of eosinophilic granulomatous gastrointestinal (GI) abscesses [16-19]. GI basidiobolomycosis affects the colon or rectum in more than $80 \%$ of cases, but small intestinal or liver involvement are each found in $30 \%$ of patients [19].

The liver fluke, F. hepatica, is one of the most widespread of all parasites that can be transmitted to humans [20]. It is found on all continents except Antartica, and is a major veterinary problem with an important economic impact on the animal reservoirs of sheep, goats, and cattle [20-22]. Since the 1980s, human infections have been increasingly identified with highly endemic areas being recognized in the American Andes, the Nile Delta, and Iran [20-26]. Outside of these endemic regions, human fascioliasis is uncommon, but outbreaks have been reported from Portugal and France [25,27]. $F$. hepatica infestations have an acute and a chronic phase [28]. In the acute stage, immature flukes penetrate the small intestinal wall and migrate into the liver. Most of these immature organisms are destroyed, but after 3-4 months, those that survive mature into adult flukes and inhabit the biliary tract where they can live for more than a decade [28].

GI basidiobolomycosis is a rare but increasingly recognized infection caused by the fungus Basidiobolus ranarum [16]. Vikram et al. [19] reviewed the worldwide cases reported through March 2010 and identified 44 patients diagnosed with GI basidiobolomycosis. The largest numbers of cases came from the desert climates of Saudi Arabia (11 patients) and Arizona in the Southwestern United States (17 patients), but four patients each were reported from Brazil and Iran and single patients from Nigeria, India, Bangladesh, Italy, the Netherlands, and an unknown country [16-19]. Recently, 14 additional patients were reported from Shiraz, Iran, a city with a climate and geography similar to Sulaimaniyah
[29]. The fungus occurs worldwide, and in the tropics, subcutaneous eosinophilic abscesses due to the traumatic inoculation of basidiobolomycosis are well recognized [16]. The source of the GI infections is not known, but pica and eating unwashed vegetables have been suggested as predisposing factors [30].

Sulaimaniyah is the provincial capital of the eastern region of Northern Iraq. Since the 2003 Gulf War, the city has grown rapidly and has incorporated pastoral and agricultural lands where cattle, sheep, and goats are raised together with fruit and vegetable crops in close proximity to newly developed neighborhoods. The mountain highlands of Northern Iraq have a semi-arid summer climate much like the Southwestern United States but with cool, wet winters similar to endemic areas of $F$. hepatica in the Andes [28].

Because deep eosinophilic granulomatous abscesses have different causes according to the part of the world from which they are reported, a cluster of such abdominal abscesses occurring over a period of 40 months in Sulaimaniyah, Iraq were investigated by serological and tissue studies to identify possible infectious agents. The findings are presented to provide physicians, surgeons, and pathologists an awareness of deep eosinophilic abscesses and to report our etiologic findings from this region of the Middle-East.

\section{Methods}

The Sulaimaniyah Directorate of Health serves as the ethics committee for Shoresh General Hospital and gave permission for the research. The research was conducted according to the Helsinki Accords. Written informed consent was obtained from patients and, in the case of children, from parents for participation in the study. Consent was granted for using personal information and specimen and radiographic images as case reports.

The study is a clinicopathological evaluation of patients in which eosinophilic granulomatous inflammation not attributable to Langerhans cell disease was recognized in surgical pathology specimens. From May 2009 to August 2012, fourteen patients were identified by pathologists ( $\mathrm{HAH}, \mathrm{RAM}$, and $\mathrm{MDH}$ ) as having liver or gastrointestinal abscesses composed of eosinophilic granulomatous inflammation. The surgical pathology specimens were received in $10 \%$ formaldehyde in the pathology departments of the two major Sulaimaniyah hospitals, Shorsh General Hospital and the Teaching Hospital of the University of Sulaimaniyah. None of the tissue was received fresh and neither the pathologists nor the surgeons submitted any specimens for bacterial or fungal cultures. The gross specimens of liver were crosssectioned at 3-4 mm intervals and examined grossly for larvae, round worms, or flukes. For microscopy, 4-16 blocks of tissue were obtained from the formalin-fixed 
gross specimens and serial sections were trimmed from the paraffin blocks. The slides were stained with hematoxylin and eosin and periodic acid-Schiff-hematoxylin (PAS-H) stains. Microscopic slides were reviewed by all pathologists (HAH, RAM, ABF, JG, and MDH).

With the identification of the early cases, the pathology was recognized as being unusual, and beginning in May of 2010 when fungal elements were first seen, microscopic slides and paraffin blocks were sent for consultation to experts in infectious disease pathology at Emory University, Atlanta, GA (ABF, JG). One of the Atlanta pathologists (JG) had been involved in evaluating the Arizona basidiobolomycosis cases [16].

All patients had abdominal ultrasound examinations, and 9 of the 14 patients had abdominal computerized tomography (CT). Three patients with abdominal abscesses (patients 1, 3, and 5) had endoscopy and endoscopic colon biopsies before laparotomy and surgery. A standardized questionnaire was developed, and patients were interviewed about symptoms and their time of onset, place of residence, type of employment, diet, contact with animals, and sources of food. Interviews were conducted after the histopathologic diagnosis were made, and after May 2010, before the initiation of anti-fungal or antiparasitic therpy. Prior to May of 2010, we did not have kits for serological testing, and based upon our review of the literature, three patients $(7,13$, and 14) were suggested to have Toxocara infestations and were treated with albendazol. They were later recalled for serologic tests.

At the time of the interviews, preoperative medical records were reviewed, blood samples were collected for completely blood counts and serological studies, and stool samples were examined microscopically for ova and parasites with emphasisis on the ova of Fasciola. Enzyme Linked Immunoabsorbent Assays (ELISA) were performed using commercial kits for F. hepatica (Pishtaz Teb Zaman ${ }^{\mathrm{TM}}$ Diagnostics, Tehran, Iran), Echinococcus (Pishtaz Teb Zaman ${ }^{\text {TM }}$ Diagnostics, Tehran, Iran), and Toxocara (Diagnostic Automation ${ }^{\mathrm{TM}}$, Inc, Calabasa, CA). All kits detected IgG antibodies to the indicated parasites using solid phase excretory-secretory (ES) antigens. All tests were performed in duplicate with positive and negative controls.

Irbil and Dohuk are the other large cities in the Kurdish region that have medical reference centers. Irbil lies in the center of the region and Duhuk in the West near the Syrian border. $\mathrm{HAH}, \mathrm{RAM}$, and $\mathrm{MDH}$ have had several discussions with the pathologists at the Hawler University Teaching Hospitals in Irbil and the Teaching Hospital of Dohuk University about whether they have encountered similar cases in their Directorates with the latest queries being by telephone in December of 2012

To find a possible source of the organisms, cattle were investigated at the regional slaughterhouse in October
2010 by the senior consultant pathologist of Shorsh Hospital $(\mathrm{MDH})$. The livers, biliary tracts, and upper gastrointestinal tracts of cattle processed during one day of work were examined by serially cross sectioning the livers and opening the duodenums and common bile ducts.

\section{Results}

A total of 14 patients (nine males and five females) were identified as having eosinophilic granulomatous abdominal abscesses. Two of the males were 18 month old children. The median age of the adults was 36 with a range of 17 to 59 years old. Summaries of patient clinical, radiological, and laboratory characteristics are provided in Table 1 and pathology, treatment, and follow-up information in Table 2. None of the patients were diabetic or were being treated with corticosteroids or other immunosuppressive drugs. HIV infections are very rare in the Kurdish region, and none of the patients had any HIV risk factors or had traveled outside of Iraq. Stools were negative for ova and parasites.

Patient 1 lived in a rural mountain village to the North of Sulaimaniyah; all other patients lived in urban Sulaimaniyah and all had visited rural areas multiple times within the year prior to diagnosis. Only patient 5 had any significant previous disease and had received ongoing treatment for chronic gastritis with esomeprazole beginning at age 25 . None of the patients had occupations related to farming, produce distribution, or animal care. All patients ate market vegetables that were washed with water or light soap or salt solutions. Vinegar or potassium permanganate was not used for washing vegetables by any of the patient's families.

Six patients had GI ulcers that in four patients produced tumor-like cecal and ascending colon masses composed of eosinophilic abscesses (Figure 1). The abscesses had irregularly shaped necrotic centers containing eosinophils with variable numbers of neutrophils (Figure 2). The margins of the abscesses were lined by a granulomatous reaction consisting of epithelioid histiocytes and Langerhan's giant cells. Eosinophil crystals were occasionally but not commonly seen. The abscesses and surrounding inflammation contained broad pauci-septate hyphae surrounded by eosinophilic material producing the Splendore-Hoeppli phenomenon (Figure 3). The hyphae were PAS positive and morphologically resembled the Zygomycete fungus Basidiobolus ranarum and were consistent with a diagnosis of probable basidiobolomycosis (Figure 4). These patients included a 39 year old man with ulcerating eosinophilic granulomatous inflammation that produced extensive soft tissue infiltration that narrowed the nasopharynx and involved the oral cavity including the floor of the mouth, base of the tongue, supraglottis, and glottis (Figure 5). The severe stenosis of the laryngopharynx required a 
Table 1 Clinical, radiologic, and laboratory characteristics of 14 patients diagnosed with granulomatous and eosinophilic gastrointestinal and hepatic abscesses in Sulaimaniyah, Iraq, 2009-2012

\begin{tabular}{|c|c|c|c|c|c|c|c|c|c|c|}
\hline Patient & $\begin{array}{l}\text { Age } \\
\text { (yrs) }\end{array}$ & Sex & $\begin{array}{l}\text { Date (mo/yr) } \\
\text { and method of } \\
\text { diagnosis }\end{array}$ & $\begin{array}{l}\text { Social and } \\
\text { medical } \\
\text { history }\end{array}$ & Febrile & $\begin{array}{l}\text { Weight } \\
\text { loss }\end{array}$ & $\begin{array}{l}\text { Eosino- } \\
\text { philia }\end{array}$ & $\begin{array}{l}\text { Principal } \\
\text { symptoms/ } \\
\text { duration }\end{array}$ & Radiologic findings & $\begin{array}{l}\text { Fasciola } \\
\text { ELISA }\end{array}$ \\
\hline 1. & 48 & M & $\begin{array}{l}\text { 5/2010; } \\
\text { histology }\end{array}$ & $\begin{array}{l}\text { Cashier/ } \\
\text { healthy }\end{array}$ & Yes & Yes & $12 \%$ & $\begin{array}{l}\text { Abdominal pain/2 } \\
\text { mos, intestinal } \\
\text { mass, obstruction }\end{array}$ & $\begin{array}{l}\text { CT: Cecal, ascending colon } \\
\text { mass, stricture. Rt. lobe liver } \\
\text { mass }\end{array}$ & Neg \\
\hline 2. & 1.5 & M & $\begin{array}{l}8 / 2010 ; \\
\text { histology, } \\
\text { biopsy of pelvic } \\
\text { mass }\end{array}$ & $\begin{array}{l}\text { Previously well } \\
\text { infant }\end{array}$ & Yes & Yes & $29 \%$ & $\begin{array}{l}\text { Abdominal pain, } \\
\text { pelvic mass/1 wk. }\end{array}$ & $\begin{array}{l}\text { CT: Necrotic mass, lower } \\
\text { abdomen and pelvis }\end{array}$ & Neg \\
\hline 3. & 59 & M & $\begin{array}{l}\text { 10/2010; } \\
\text { histology }\end{array}$ & $\begin{array}{l}\text { Merchant, dry } \\
\text { goods/healthy }\end{array}$ & Yes & $\begin{array}{l}\text { Yes } \\
(6 \mathrm{~kg})\end{array}$ & No & $\begin{array}{l}\text { Abdominal pain } \\
\text { and mass. Itching } 3 \\
\text { mos. }\end{array}$ & CT: Cecal mass & Neg \\
\hline 4. & 53 & M & $\begin{array}{l}\text { 2/2012; } \\
\text { histology }\end{array}$ & $\begin{array}{l}\text { Soldier/ } \\
\text { healthy }\end{array}$ & Yes & Yes & $\begin{array}{l}\text { Not } \\
\text { tested }\end{array}$ & $\begin{array}{l}\text { Abdominal pain } / 1 \\
\text { mo., intestinal mass, } \\
\text { obstruction }\end{array}$ & $\begin{array}{l}\mathrm{CT} \text { : Cecal, transverse colon } \\
\text { masses, strictures }\end{array}$ & Neg \\
\hline 5. & 39 & M & $\begin{array}{l}\text { 5/2012; } \\
\text { histology }\end{array}$ & $\begin{array}{l}\text { Military office } \\
\text { worker/ } \\
\text { chronic } \\
\text { gastritis }\end{array}$ & Yes & Yes & $9 \%$ & $\begin{array}{l}\text { Persistent cough, } \\
\text { sore throat/1 mo. }\end{array}$ & $\begin{array}{l}\text { CT: Diffuse soft tissue mass } \\
\text { of oropharynx. Transverse } \\
\text { colon mass, stricture }\end{array}$ & Neg \\
\hline 6. & 1.5 & M & $\begin{array}{l}\text { 8/2012; } \\
\text { histology }\end{array}$ & $\begin{array}{l}\text { Previously well } \\
\text { infant }\end{array}$ & Yes & Yes & $21 \%$ & $\begin{array}{l}\text { Abdominal pain/2-3 } \\
\text { d, intestinal mass, } \\
\text { obstruction }\end{array}$ & $\begin{array}{l}\text { CT: Cecal mass, thick } \\
\text { ascending colon with } \\
\text { stricture }\end{array}$ & Neg \\
\hline 7. & 30 & M & $\begin{array}{l}\text { 6/2009; } \\
\text { histology, } \\
\text { serology }\end{array}$ & Janitor/healthy & Yes & Yes & $26 \%$ & $\begin{array}{l}\text { R subcostal pain } / 1 \\
\text { mo. }\end{array}$ & $\begin{array}{l}\text { CT: Cystic mass Rt. lobe liver. } \\
\text { Mild splenomegaly }\end{array}$ & Pos \\
\hline 8. & 30 & $\mathrm{~F}$ & $\begin{array}{l}\text { 5/2010; } \\
\text { histology, } \\
\text { serology }\end{array}$ & $\begin{array}{l}\text { Housewife/ } \\
\text { healthy }\end{array}$ & No & No & $>50 \%$ & $\begin{array}{l}\text { R subcostal pain } / 1 \\
\text { wk. }\end{array}$ & $\begin{array}{l}\text { US: Small hypodense liver } \\
\text { lesions. Mild splenomegaly }\end{array}$ & Pos \\
\hline 9. & 56 & $\mathrm{~F}$ & $\begin{array}{l}\text { 6/2010; } \\
\text { histology, } \\
\text { serology }\end{array}$ & $\begin{array}{l}\text { Housewife/ } \\
\text { healthy }\end{array}$ & No & $\begin{array}{l}\text { Yes } \\
(7 \mathrm{~kg})\end{array}$ & $35 \%$ & $\begin{array}{l}\text { R subcostal pain } / 1-2 \\
\text { wks. }\end{array}$ & $\begin{array}{l}\text { US: Small hypodense liver } \\
\text { lesions }\end{array}$ & Pos \\
\hline 10. & 56 & $\mathrm{~F}$ & $\begin{array}{l}8 / 2010 ; \\
\text { histology, } \\
\text { serology }\end{array}$ & $\begin{array}{l}\text { Housewife/ } \\
\text { healthy }\end{array}$ & Yes & Yes & $26 \%$ & $\begin{array}{l}\text { R subcostal pain } / 1-2 \\
\text { wks. }\end{array}$ & US: Cystic mass Rt. lobe liver & Pos \\
\hline 11. & 33 & M & $\begin{array}{l}\text { 11/2011; } \\
\text { histology, } \\
\text { serology }\end{array}$ & $\begin{array}{l}\text { Shopkeeper/ } \\
\text { healthy }\end{array}$ & Yes & $\begin{array}{l}\text { Yes }(10 \\
\mathrm{kg})\end{array}$ & $72 \%$ & $\begin{array}{l}\text { R subcostal pain } / 2-3 \\
\text { wks. }\end{array}$ & $\begin{array}{l}\text { CT: Cystic mass Rt. lobe liver. } \\
\text { Mild splenomegaly }\end{array}$ & Pos \\
\hline 12. & 22 & $\mathrm{~F}$ & $\begin{array}{l}\text { 5/2010; } \\
\text { histology, } \\
\text { serology }\end{array}$ & $\begin{array}{l}\text { Student/ } \\
\text { healthy }\end{array}$ & No & $\begin{array}{l}\text { Yes } \\
(4 \mathrm{~kg})\end{array}$ & $74 \%$ & $\begin{array}{l}\text { R subcostal pain } / 1 \\
\text { wk. }\end{array}$ & $\begin{array}{l}\text { US: Small hypodense liver } \\
\text { lesions. Mild splenomegaly }\end{array}$ & Borderline \\
\hline 13. & 17 & $\mathrm{~F}$ & $\begin{array}{l}\text { 5/2009; } \\
\text { histology }\end{array}$ & $\begin{array}{l}\text { Student/ } \\
\text { healthy }\end{array}$ & Yes & Yes & $23 \%$ & $\begin{array}{l}\text { Abdominal pain } / 2-3 \\
\text { wks. }\end{array}$ & $\begin{array}{l}\text { US: Cystic mass Rt. lobe liver. } \\
\text { Mild splenomegaly }\end{array}$ & Neg \\
\hline 14. & 32 & M & $\begin{array}{l}\text { 4/2009; } \\
\text { histology }\end{array}$ & $\begin{array}{l}\text { Soldier/ } \\
\text { healthy }\end{array}$ & No & No & $29 \%$ & $\begin{array}{l}\text { R subcostal pain/1-2 } \\
\text { mos. }\end{array}$ & CT: Cystic mass Rt. lobe liver & Neg \\
\hline
\end{tabular}

Abbreviations: $M$ male, $F$ female. Mo, month; wk, week; d, day. CT, computerized tomography; US, ultrasonography. Neg, negative; Pos, positive.

tracheostomy. Three months after the diagnosis of the oropharyngeal disease, the patient developed intestinal obstruction and was found to have an eosinophilic granulomatous mass in the transverse colon that contained hyphae with a Splendore-Hoeppli reaction. One of the patients with GI disease also had a localized liver abscess containing fungus.

Eight patients had isolated eosinophilic granulomatous liver abscesses. In 5 patients the disease was localized with a single multilocular lesion being identified radiologically and at laparotomy in the right hepatic lobe (Figure 6). Localized abscesses were resected by segmental hepatic lobectomy. In 3 patients, the disease was diffuse with many small lesions $(0.5-2 \mathrm{~cm})$ being scattered throughout the liver. The patients with diffuse small abscesses were operated on for symptoms of gall bladder disease, and underwent cholecystectomy and wedge biopsies of the liver abscesses. The gall bladders showed mild chronic cholecystitis, and by ultrasonography, the common ducts were not dilated and contained no stones or shadows. The 
Table 2 Pathological findings, treatment, and follow-up for 14 patients diagnosed with granulomatous and eosinophilic gastrointestinal and hepatic abscesses in Sulaimaniyah, Iraq, 2009-2012

\begin{tabular}{|c|c|c|c|c|c|c|c|}
\hline Patient & Surgical procedure & $\begin{array}{l}\text { Pathologic findings and } \\
\text { location of disease }\end{array}$ & $\begin{array}{l}\text { Size of } \\
\text { abscesses }\end{array}$ & $\begin{array}{l}\text { Organism in } \\
\text { tissue }\end{array}$ & $\begin{array}{l}\text { Medical } \\
\text { treatment }\end{array}$ & Complications & $\begin{array}{l}\text { Follow-up } \\
\text { duration }\end{array}$ \\
\hline 1. & $\begin{array}{l}\text { Right hemicolectomy } \\
\text { Segmental hepatectomy }\end{array}$ & $\begin{array}{l}\text { Cecal, ascending colon, and } \\
\text { localized liver masses }\end{array}$ & $8-12 \mathrm{~cm}$ & $\begin{array}{l}\text { Pauci-septate } \\
\text { hyphae }\end{array}$ & $\begin{array}{l}\text { Oral itraconozol, } \\
6 \text { mos. }\end{array}$ & $\begin{array}{l}\text { Recurrent } \\
\text { obstruction after } 5 \\
\text { mos. }\end{array}$ & $\begin{array}{l}\text { Asymptomatic, } \\
24 \text { mos. }\end{array}$ \\
\hline 2. & Biopsy of mass & $\begin{array}{l}\text { Pelvic mass, intestinal site } \\
\text { uncertain }\end{array}$ & $9 \mathrm{~cm}$ & $\begin{array}{l}\text { Pauci-septate } \\
\text { hyphae }\end{array}$ & $\begin{array}{l}\text { IV amphotercin } \\
\text { B, } 2 \mathrm{~d} \text {. }\end{array}$ & Died & \\
\hline 3. & Right hemicolectomy & Cecal mass & $6 \mathrm{~cm}$ & $\begin{array}{l}\text { Pauci-septate } \\
\text { hyphae }\end{array}$ & $\begin{array}{l}\text { Oral itraconozol, } \\
6 \text { mos }\end{array}$ & None & $\begin{array}{l}\text { Asymptomatic, } \\
26 \text { mos. }\end{array}$ \\
\hline 4. & $\begin{array}{l}\text { Right hemi and transverse } \\
\text { colectomy }\end{array}$ & $\begin{array}{l}\text { Cecal and transverse colon } \\
\text { masses }\end{array}$ & $17 \mathrm{~cm}$ & $\begin{array}{l}\text { Pauci-septate } \\
\text { hyphae }\end{array}$ & $\begin{array}{l}\text { Oral itraconozol, } \\
6 \text { mos. }\end{array}$ & $\begin{array}{l}\text { Recurrent } \\
\text { obstruction after } 3 \\
\text { mos. }\end{array}$ & $\begin{array}{l}\text { Asymptomatic, } \\
10 \text { mos. }\end{array}$ \\
\hline 5. & Transverse colectomy & $\begin{array}{l}\text { Oropharyngeal and } \\
\text { transverse colon masses }\end{array}$ & $20 \times 9 \mathrm{~cm}$ & $\begin{array}{l}\text { Pauci-septate } \\
\text { hyphae }\end{array}$ & $\begin{array}{l}\text { Itraconozol: IV14 } \\
\text { d, Oral } 7 \text { mos }\end{array}$ & None & $\begin{array}{l}\text { Asymptomatic, } \\
7 \text { mos. }\end{array}$ \\
\hline 6. & Right hemicolectomy & Cecal mass & $4 \mathrm{~cm}$ & $\begin{array}{l}\text { Pauci-septate } \\
\text { hyphae }\end{array}$ & $\begin{array}{l}\text { Oral itraconozol, } \\
4 \text { mos. }\end{array}$ & None & $\begin{array}{l}\text { Asymptomatic, } \\
4 \text { mos. }\end{array}$ \\
\hline 7. & Segmental hepatectomy & Liver mass, localized & $4 \times 6 \mathrm{~cm}$ & No & $\begin{array}{l}\text { Albendazole } \\
400 \mathrm{mg} / \mathrm{d}, 3 \mathrm{~d}\end{array}$ & None & $\begin{array}{l}\text { Asymptomatic, } \\
41 \text { mos. }\end{array}$ \\
\hline 8. & $\begin{array}{l}\text { Cholecystectomy, wedge } \\
\text { liver biopsy }\end{array}$ & Liver, diffuse abscesses & $0.5-2 \mathrm{~cm}$ & No & $\begin{array}{l}\text { Albendazole } \\
400 \mathrm{mg} / \mathrm{d}, 3 \mathrm{~d}\end{array}$ & None & $\begin{array}{l}\text { Asymptomatic, } \\
30 \text { mos. }\end{array}$ \\
\hline 9. & $\begin{array}{l}\text { Cholecytectomy, wedge } \\
\text { liver biopsy }\end{array}$ & Liver, diffuse abscesses & $0.5-2 \mathrm{~cm}$ & No & $\begin{array}{l}\text { Albendazole } \\
400 \mathrm{mg} / \mathrm{d}, 3 \mathrm{~d}\end{array}$ & $\begin{array}{l}\text { Common duct } \\
\text { fluke after } 2 \text { yrs }\end{array}$ & $\begin{array}{l}\text { Asymptomatic, } \\
30 \text { mos. }\end{array}$ \\
\hline 10. & Segmental hepatectomy & Liver mass, localized & $3 \mathrm{~cm}$ & No & $\begin{array}{l}\text { Albendazole } \\
400 \mathrm{mg} / \mathrm{d}, 3 \mathrm{~d}\end{array}$ & None & $\begin{array}{l}\text { Asymptomatic, } \\
28 \text { mos. }\end{array}$ \\
\hline 11. & Segmental hepatectomy & Liver mass, localized & $5 \mathrm{~cm}$ & No & $\begin{array}{l}\text { Albendazole } \\
400 \text { mg/d, } 3 \text { d }\end{array}$ & None & $\begin{array}{l}\text { Asymptomatic, } \\
13 \text { mos. }\end{array}$ \\
\hline 12. & $\begin{array}{l}\text { Cholecytectomy, wedge } \\
\text { liver biopsy }\end{array}$ & Liver, diffuse abscesses & $0.5-2 \mathrm{~cm}$ & No & $\begin{array}{l}\text { Albendazole } \\
400 \mathrm{mg} / \mathrm{d}, 3 \mathrm{~d}\end{array}$ & None & $\begin{array}{l}\text { Asymptomatic, } \\
30 \text { mos. }\end{array}$ \\
\hline 13. & Segmental hepatectomy & Liver mass, localized & $4 \times 5 \mathrm{~cm}$ & No & $\begin{array}{l}\text { Albendazole } \\
400 \mathrm{mg} / \mathrm{d}, 3 \mathrm{~d}\end{array}$ & None & $\begin{array}{l}\text { Asymptomatic, } \\
42 \text { mos. }\end{array}$ \\
\hline 14. & Segmental hepatectomy & Liver mass, localized & $2 \mathrm{~cm}$ & No & $\begin{array}{l}\text { Albendazole } \\
400 \text { mg/d, } 3 \text { d }\end{array}$ & None & $\begin{array}{l}\text { Asymptomatic, } \\
43 \text { mos. }\end{array}$ \\
\hline
\end{tabular}

isolated liver abscesses, both localized and diffuse, consisted of epithelioid and mutinucleated histiocytes surrounding irregular central zones of necrotic neutrophils and eosinophils with prominent elongate or rhomboid eosinophil crystals (Figure 7). Neither larvae suggesting Toxocara nor Fasciola flukes or ova were found in any of the liver specimens.

Five of the 8 patients with isolated liver abscesses tested serologically positive for $F$. hepatica, one had a borderline result, and two were negative. The two negative patients had their disease identified before May of 2010 and had been treated with albendazol before having blood drawn for serological testing 14 and 15 months after diagnosis. One serologically positive patient had blood drawn 13 months after being treated. All six patients with probable basidiobolomycosis had negative fasciola ELISA results. All 14 patients were serologically negative for Toxocara and Echinococcus.

The adults and one child with GI basidiobolomycosis

were treated by right hemicolectomy and/or transverse
Figure 1 Patient 1. A right hemicolectomy specimen showing a cecal mass with irregular mucosal ulceration. 


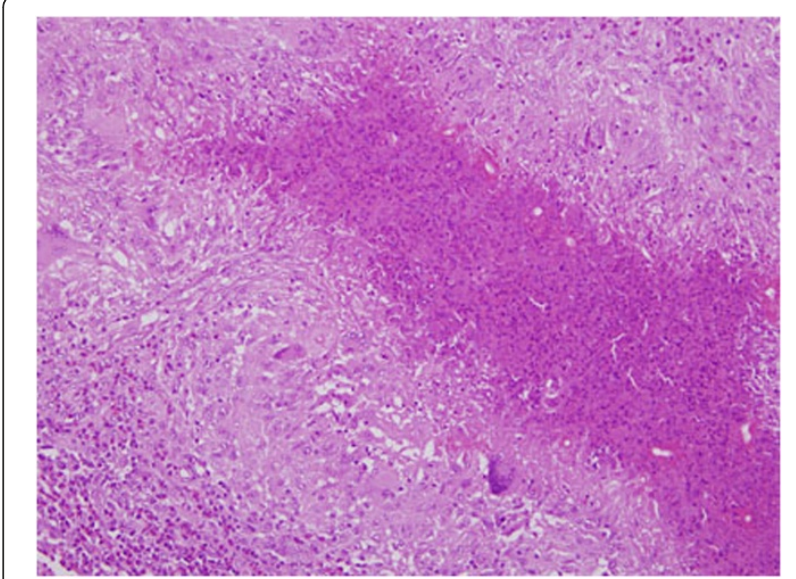

Figure 2 Patient 1. The wall of the cecum demonstrates a stellate abscess with a necrotic center containing eosinophils surrounded by granulomatous inflammation with Langerhans giant cells. Fungal elements can be seen surrounded by an eosinophilic SplendoreHoeppli reaction. Hematoxylin and eosin stain $\times 100$.

colectomy for the GI masses, and for the patient with liver involvement by segmental hepatectomy. Surgery was followed by oral itraconozol at $400 \mathrm{mg} /$ day for 4 to 7 months. Two adults required additional surgery for recurrent intestinal obstruction, one at 3 months and the other at 5 months following the original colonic resection. One of the children (patient 2) developed intestinal perforation and died while he was receiving intravenous amphotericin B. Intravenous itraconozol was not available, and the child was too ill to undergo extensive surgery at the time basidiobolomycosis was diagnosed on the basis of an incisional biopsy obtained at laparotomy from the outer aspect of a large pelvic mass. Intravenous itraconozole was obtained for the patient with oropharyngeal disease. After

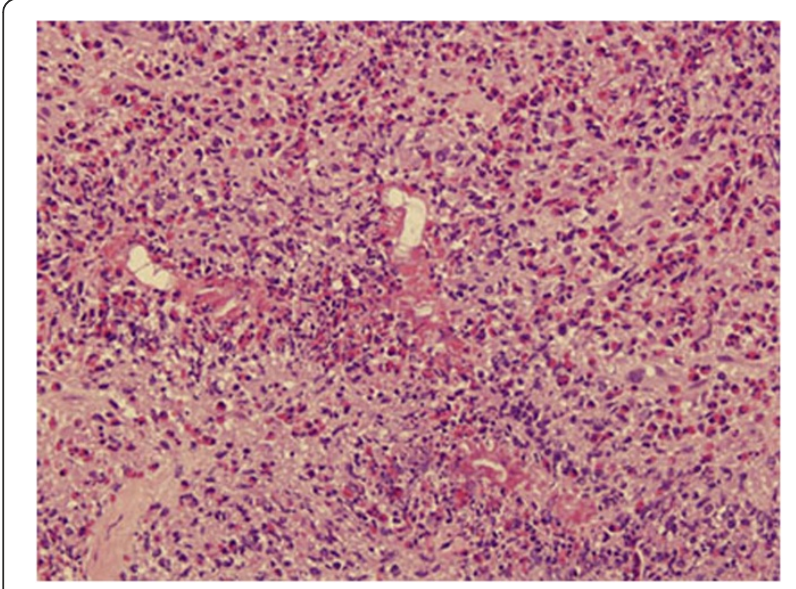

Figure 3 Patient 5. The microscopy of a tonsilar biopsy reveals an intense eosinophilic inflammatory reaction containing fungal hyphae surrounded by the Splendore-Hoeppli phenomenon. Hematoxylin and eosin stain $\times 200$

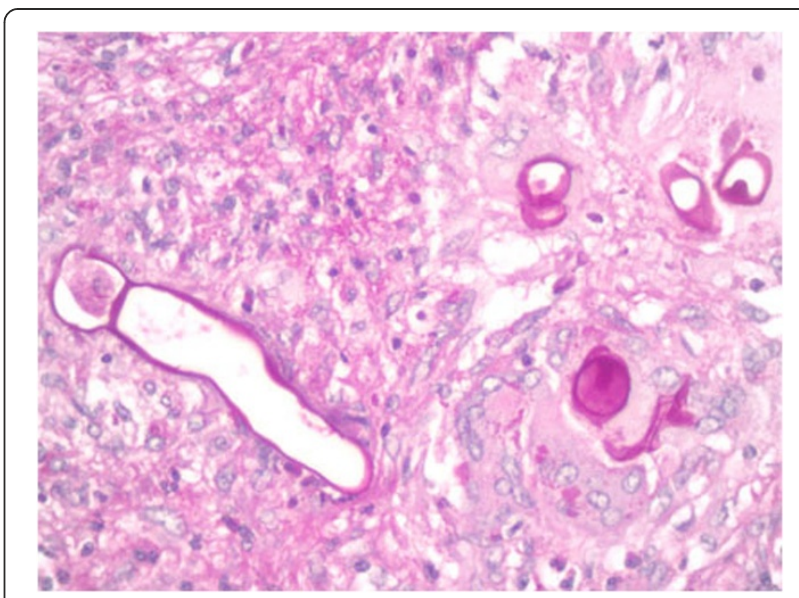

Figure 4 Patient 1. The fungi consist of broad pauci septate hyphae consistent with basidiobolomycosis. Periodic acid-Schiff hematoxylin stain $\times 400$.

two months, the tissues of the oropharynx decreased in size, and airway and esophageal access were restored. The infected segment of transverse colon, that was found three months after beginning treatment of the oropharyngeal disease, was resected, and the patient continued 7 months of oral itraconozle with no further complications.

After surgery, patients with isolated liver abscesses including those with positive, borderline, and negative fasciola ELISA results were treated with a 3 day course of $400 \mathrm{mg}$ daily of albendazole. Constitutional symptoms resolved within the 3 day period of treatment and eosinophilia within one to 3 months afterward. Two years after her initial treatment, patient 10 developed right

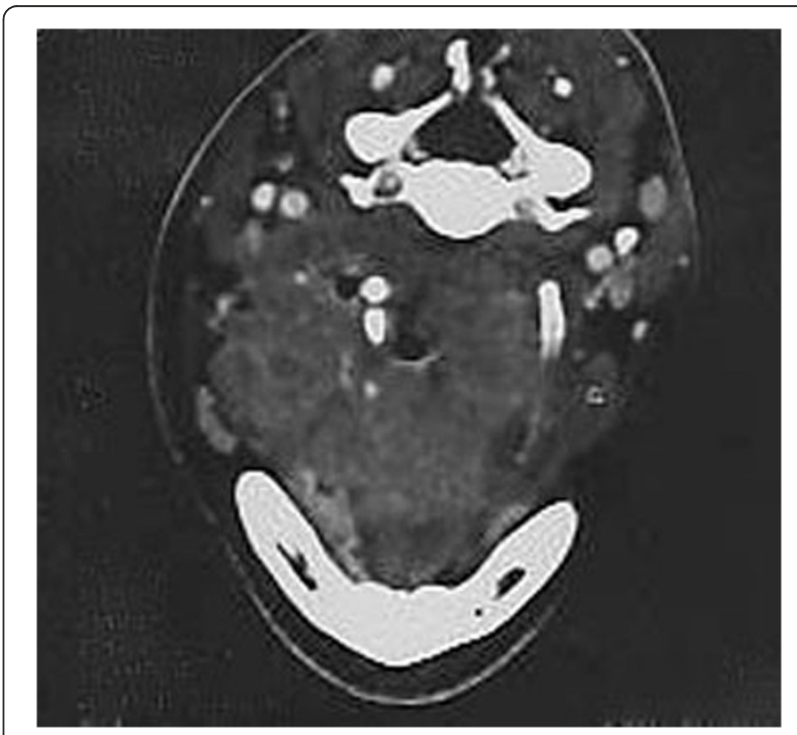

Figure 5 Patient 5. Magnetic resonance imaging showing a mass at the base of the tongue that markedly narrows and shifts the oropharynx laterally. 


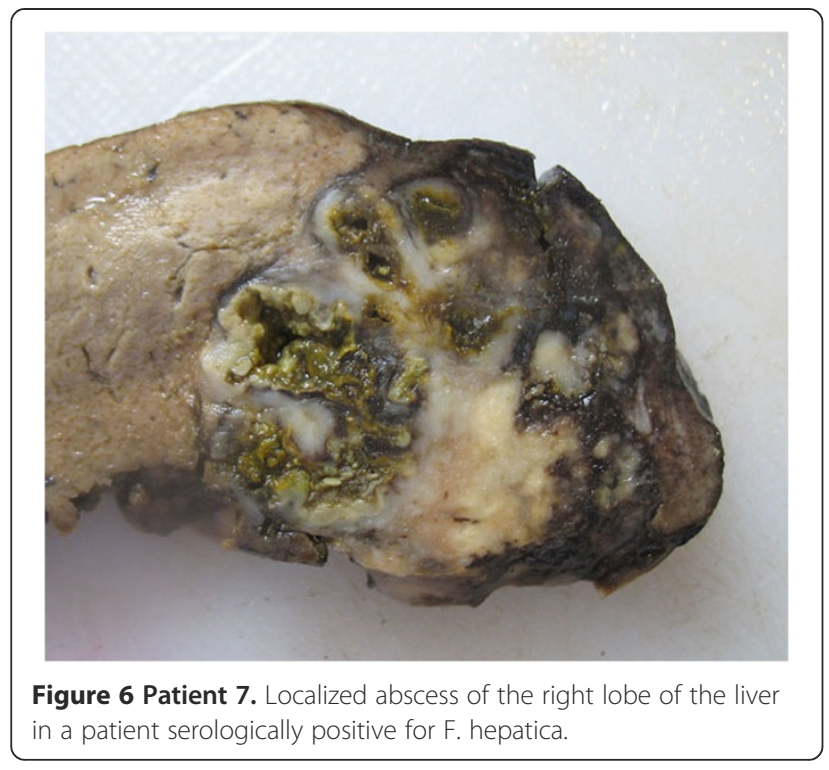

upper quadrant pain and tenderness and was found to have a dilated common duct with a moving shadow by ultrasonography. A fluke was extracted from her common bile duct by ERCP. She was then given a single dose of $500 \mathrm{mg}$ of triclabendazole and has since been asymptomatic and has had no eosinophilia.

Most of the patients came to medical attention during the warmer and dryer months of the year. The patients with probable basidiobolomycosis were diagnosed in February, May (2 patients), August (2 patients), and October. Patients serologically positive or borderline for F. hepatica were diagnosed in May (one positive, one borderline), June (2 patients), August, and November.

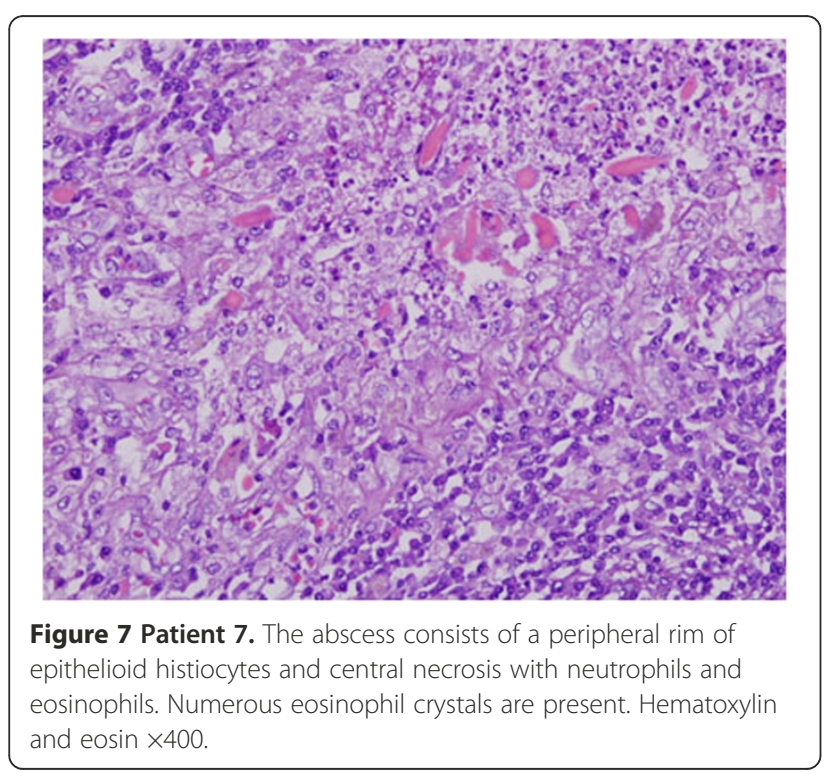

The two patients with isolated liver abscesses and negative fasciola serology were diagnosed in April and May.

Since beginning the study, we have had one case of cutaneous chromoblastomycosis and two cases of aspergillus allergic fungal sinusitis, but have not seen any eosinophilic subcutaneous abscesses nor any subcutaneous or sinus fungal infections consistent with entomophthoromycosis. We have had one case of invasive sino-nasal mucormycosis in a diabetic. Cancer chemotherapy patients are usually investigated by bronchial washings or fine needle aspiration cytology rather than biopsies for pulmonary infections. With this limitation, we have had only two bronchial washes containing Candida as potential fungal pulmonary infections. Endoscopic colon biopsies before laparotomy on patients 1,3 , and 5 showed eosinophilic granulomatous inflammation; fungi were seen only in the biopsy of patient 5. One eosinophilic liver abscess was reported at the Teaching Hospital of Duhuk University in 2010; none have been seen by the pathologists at Hawler University Teaching Hospitals in Erbil.

Thirty seven cattle were examined at the Sulaimaniyah slaughterhouse and $F$. hepatica was identified in ten animals (27\%). Immature flukes were found in the duodenums, and adult flukes were identified in thickened intrahepatic bile ducts (Figure 8). None of the cattle showed any evidence of fungal disease.

\section{Discussion}

In this article, we report from Northern Iraq a cluster of GI and hepatic eosinophilic granulomatous abscesses that were not recognized in the region prior to 2009 . This pathology is relatively rare, and the medical literature is vague about possible causative agents. We show that, in this region of Iraq, some eosinophilic granulomatous abscesses can be attributed to $F$. hepatica and others to a fungus consistent with Basidiobolus ranarum. Although, two of the 14 cases could not be ascribed to a specific etiologic agent, none of the patients had serological or pathological evidence for Toxocara or Echinococcus granulosis, the latter being the most common clinical parasitic disease of the region [31,32].

The currently recommended criteria for the diagnosis of fascioliasis include peripheral eosinophilia and positive serological testing for antibodies to the organism $[33,34]$. Additional symptoms of the acute hepatic stage consist of fever, malaise, right subchondral pain, and weight loss. CT may show irregular hypodense lesions within the liver [35]. Stool examinations for eggs can be diagnostic but are usually negative because of a generally low concentration of adult flukes and irregular shedding of eggs [34]. The immature flukes that penetrate the 


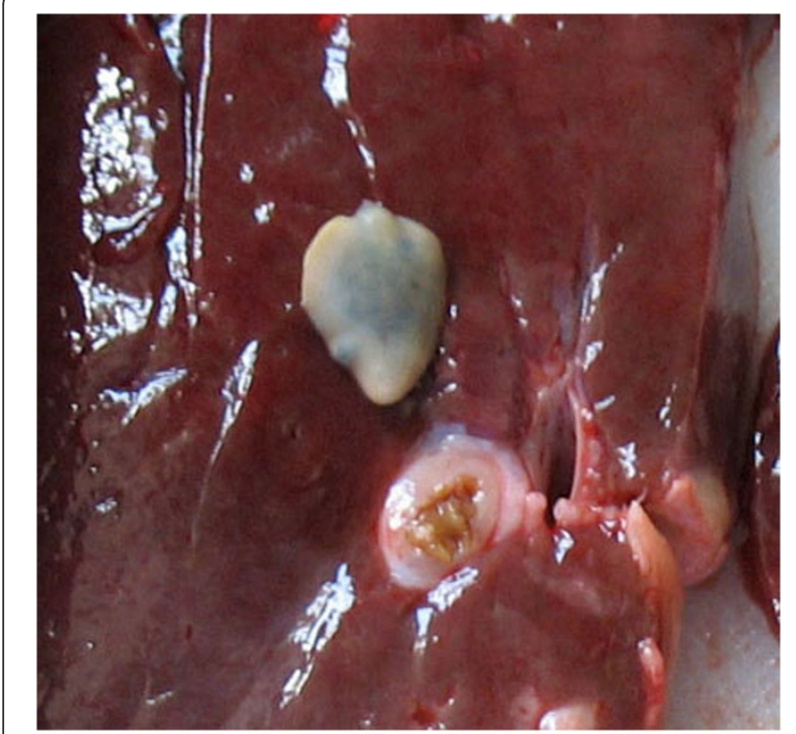

Figure 8 Liver of a bull slaughtered at the regional abattoir showing an adult fluke of $F$. hepatica that was found in the thickened bile duct below the parasite.

liver are rarely seen in histologic sections, and a tissue diagnosis of fascioliasis is usually not possible $[15,28]$.

On the basis of the serological findings, five of the 14 patients with eosinophilc abscesses were diagnosed as having $F$. hepatica infestations. On the basis of a borderline serological result, one additional patient was considered to be suspicious for fascioliasis. All 6 of these patients presented with right subcostal abdominal pain and eosinophilia. By CT or ultrasonography, a 3 to $6 \mathrm{~cm}$ in diameter cystic mass was found in the right lobe of the liver in 3 patients, and small hypodense lesions were scattered thoughout the liver in 3 other patients. The surgical pathology specimens of both the larger and the smaller lesions demonstrated eosinophilic granulomatous abscesses with no evidence of flukes or ova.

In six patients, the eosinophilic abscesses contained pauci-septate hyphae surrounded by a SplendoreHoeppli reaction. While a definitive diagnosis requires culture, a probable diagnosis of basidiobolomycosis can be made histologically [30]. The species Basidiobolus ranarum is a Zygomycete fungus belonging to the order Entomophthorales [36]. The differential diagnosis consists of the entomophthoromycosis Conidiobolus coronatus and Conidiobolus incongruous that like basidiobolomycosis incites an eosinophilic granulomatous tissue reaction and the Splendore-Hoeplli phenomonon. Conidiobolus is primarily a tropical disease that produces sinonasal infections and subcutaneous abscesses. Oropharyngeal and laryngeal disease has been reported, but Conidiobolus is not a known cause of intestinal infections [36]. Vikram et al. [19] have recommended that the definition of probable GI basidiobolomycosis include the identification of disease at a site below the esophagus in the stomach, large or small intestine, anus, liver, gall bladder, or pancreas.

In this current study, patients with probable basidiobolomycosis had fever, malaise, weight loss, and mild to moderate eosinophilia, but the predominant finding was an abdominal mass with intestinal obstruction. One patient had an ulcerating oropharyngeal and glottic mass and obstructing disease of the transverse colon. This oropharyngeal disease was included as an example of GI basidiobolomycosis on the basis of the patient's colon involvement and on the basis of the definition of the GI tract by the National Library of Medicine-Medical Subject Headings including all derivatives of the embryonic entoderm from the mouth to the anus [37]. As an example of probable oropharyngeal basidiobolomycosis, the case extends the reported level of GI involvement to above the stomach and overlaps with locations infected by Conidiobolus [19,36].

It is suggested that GI basidiobolomycosis is acquired by the ingestion of contaminated soil or animal fecal material [16]. The animal herds at the outskirts of Sulaimaniyah make dung ubiquitous, and the dry climactic conditions throughout the warm months of the year may contribute to the windborne spread of organisms [16]. It is notable that five of the six patients with basidiobolomycosis were diagnosed in May, August and October, and that currently GI basidiobolomycosis is the most common deep fungal infection diagnosed in our community. It is also notable that cutaneous entomophthoromycosis, that is rare in arid climates where the great majority of GI basidiobolomycosis is reported, has not been seen in Sulaimaniyah.

In past years, sporadic cases of the biliary phase of fascioliasis have been recognized in the Sulaimaniyah governate, and in the autumn of 2007, one of the authors (TAH) conducted a study of local streams and identified $F$. hepatica rediae in $4 \%$ of Lymnaeid snails [38]. Rediae develop into tadpole-like cercariae that leave the snail and encyst as metacercariae on vegetation in or near streams and rivers [24,27,39]. Naturally growing watercress is sold in Sulaimaniyah markets. It is a favourite vegetable for human consumption with both wild and commercial products being common sources of infection [27,39]. Studies of hyperendemic fascioliasis in the South American Andes indicate that an increased risk of livestock infection and human transmission can be anticipated when greater numbers of cattle become concentrated around limited water sources [24]. As part of our current study, we found that $27 \%$ of cattle being processed at the regional slaughter house were infected with $F$. hepatica. The growth of Sulaimaniyah into areas still used for livestock may have 
exacerbated a generally low endemic level into one exposing increased numbers of humans to infection.

The acute phase of Fasciola hepatica responds well to a single course of triclabendazole [40]. Although triclabendazole has not been available in our pharmacies, symptoms and eosinophilia resolved with a 3 day course of $400 \mathrm{mg}$ of albendazole. Nevertheless, one of the patients treated with albendazole entered a chronic biliary phase of the disease two years after the initial diagnosis. Fatal fascioliasis occurs in livestock that are infected with large numbers of organisms, but this is extremely rare in humans for whom the principal manifestations are a prolonged low-grade febrile illness with some degree of anemia and hepatomegaly $[28,41]$

The reported cases of GI basidiobolomycosis nearly all had large or small intestinal obstruction that required surgery $[19,29]$. The recommended treatment is surgical removal of the infected tissues and an imidazole as antifungal agent with itraconozole being the most frequently recommended $[16,19,36]$. The organism tends to be resistant to amphotericin B $[16,19,36]$. In the review by Vikram et al. [19], eight deaths occurred among fourfour patients (18\%) with five of the fatalities being children [16-19,42-44]. Common features of the fatal cases were intestinal perforation and/or extensive abdominal spread of the disease $[18,43]$. Of the patients who died, only one was treated with itraconozole, 4 received no anti-fungal therapy, and 3 patients an amphotericin product. In our fatal case, the patient received amphotericin B as an anti-fungal agent and died as a result of intestinal perforation before the infected tissue could be resected.

The clinicopathologic findings for the two patients in which no diagnosis could be established were similar to those diagnosed with fascioliasis. The patients had isolated hepatic abscesses, abdominal pain, and eosinophila. Both patients were treated with albendazole for presumed toxocariasis more than a year before they were tested serologically. Following eradication of organisms, the ELISA serology for Fasciola is reported to become negative in $40 \%$ of patients at 6 months and more than $90 \%$ of patients after 12 months [45]. In contrast, anti-Toxocara IgG antibodies in patients treated for visceral toxocariasis usually remain elevated for years [46,47]. We recognize that an organism other than F. hepatica may have caused the two etiologically undiagnosed cases, but with the negative serology and inability to find larvae in the abscesses, we believe that a role for Toxocara has been reasonably

Table 3 Pathological and clinical laboratory findings in the differential diagnosis of abdominal eosinophilic granulomatous abscesses

\begin{tabular}{|c|c|c|c|c|}
\hline & Basidiobolomycosis & Fascioliasis & Toxocariasis & References \\
\hline \multicolumn{5}{|l|}{$\begin{array}{l}\text { Pathologic } \\
\text { findings }\end{array}$} \\
\hline \multicolumn{5}{|l|}{$\begin{array}{l}\text { Anatomic site } \\
\text { involved }\end{array}$} \\
\hline $\begin{array}{l}\text { Intestine/colon } \\
\text { mass }\end{array}$ & $80 \%$ of patients & Rare, ectopic & Not reported & {$[16,19,46,48,49]$} \\
\hline Liver abscesses & Localized, $30 \%$ of patients & $\begin{array}{l}\text { Diffuse more common than } \\
\text { localized. }\end{array}$ & $\begin{array}{l}\text { Diffuse with VLM, localized } \\
\text { uncommon }\end{array}$ & {$[9-11,13-15,19,28,35,46]$} \\
\hline \multicolumn{5}{|l|}{$\begin{array}{l}\text { Microscopic } \\
\text { findings }\end{array}$} \\
\hline $\begin{array}{l}\text { Granulomatous } \\
\text { inflammation }\end{array}$ & Present & Present & Present & {$[12-16,19,28]$} \\
\hline Eosinophils & Present & Present & Present & {$[12-16,19,28]$} \\
\hline $\begin{array}{l}\text { Charcot-Leyden } \\
\text { crystals }\end{array}$ & Not prominent & Numerous & Present & {$[12-16,19,28]$} \\
\hline $\begin{array}{l}\text { Organisms in } \\
\text { tissues }\end{array}$ & $\begin{array}{l}\text { Pauci-septate hyphae with Splendore- } \\
\text { Hoeppli reaction required for diagnosis }\end{array}$ & $\begin{array}{l}\text { Very rare, if present ova more } \\
\text { common than flukes }\end{array}$ & Larvae $30 \%$ of cases & {$[12-16,19,28,46,50]$} \\
\hline \multicolumn{5}{|l|}{$\begin{array}{l}\text { Clinical laboratory } \\
\text { findings }\end{array}$} \\
\hline Eosinophilia & $76 \%$ of patients, usually not very high & $\begin{array}{l}\text { Consistently positive in acute } \\
\text { stage }\end{array}$ & $\begin{array}{l}\text { Consistently positive with } \\
\text { VLM }\end{array}$ & {$[12-16,19,28,46,47,50]$} \\
\hline \multicolumn{5}{|l|}{ Serology } \\
\hline $\begin{array}{l}\text { Before } \\
\text { treatment }\end{array}$ & $\begin{array}{l}\text { Immunodiffusion positive } 50 \% \text { of } \\
\text { patients, not routinely available. }\end{array}$ & $\begin{array}{l}\text { ELISA } 87-93 \% \text { sensitivity, } 98 \% \\
\text { specificity for acute stage }\end{array}$ & $\begin{array}{l}\text { ELISA } 78 \% \text { sensitivity, } 98 \% \\
\text { specificity for VLM }\end{array}$ & {$[16,19,33,47,50]$} \\
\hline After treatment & Unknown & $>90 \%$ negative at 1 year. & Positive for years after VLM & {$[16,19,33,45-47]$} \\
\hline
\end{tabular}


ruled out and that these patients may have had fascioliasis with an attenuated antibody response.

In order to reach a correct diagnosis and determine the cause of an eosinophilic granulomatous abdominal abscess, pathologists, surgeons, and physicians should be aware of the different possible etiologies. A presentation as a GI mass must be distinguished from cancer, and the granulomatous inflammation surrounding the central abscess can be mistaken for tuberculosis. We have seen the eosinophilia of fascioliasis erroneously treated as an idiopathic hypereosinophilia syndrome with imatinib mesylate.

The fungus of basidiobolomycosis is readily identified if sufficient tissue is obtained but can be missed in mucosal biopsies as it was in two of our patients. The diagnosis of fascioliasis has historically been a problem. Serological testing is often unavailable, or an ineffective method may used. Before 2003, the sensitivity of serological tests was poor, but newer ELISA kits using ES or Fas2 antigens have an estimated sensitivity of $87-93 \%$ and are currently the recommended methods for diagnosing $F$. hepatica $[33,34,40,45]$.

The gastrointestinal location of an eosinophilic granulomatous mass points to basidiobolomycosis, but rare examples of ectopic fascioliasis producing tumor-like colonic masses are reported $[28,48,49]$. In addition, hepatic abscesses are found in a significant proportion of patients with basidiobolomycosis [19]. It is not the location of eosinophilic granulomatous disease, but the identification of an Entomophthorales fungus that provides for the diagnosis of basidiobolomycosis, and the absence of fungus and a positive serological test for Fasciola that allows for the diagnosis of fascioliasis. Table 3 summarizes the pathological and serological findings for basidiobolomycosis, fascioliasis, and toxocariasis, the latter being included because it is considered a frequent cause of eosinophic granulomatous liver abscesses [9-11,13,46,50].

The identification of disease at surgery uncovers only the most severely affected patients. Serological studies for F. hepatica in the urban and rural populations of Sulaimaniyah as well as the examination of livestock are currently in progress to determine the level of the endemic situation. The clustering of basidiobolomycosis and F. hepatica has not been previously reported. The epidemiology of $F$. hepatica has been thoroughly studied but factors predisposing to basidiobolomycosis are poorly understood [16,20-25,30]. We believe Sulaimaniyah may provide an opportunity to identify conditions that contribute to human outbreaks of both diseases.

\section{Conclusions}

Eosinophilic granulomatous abscesses unrelated to Langerhans histiocytosis are relatively rare human diseases of often obscure etiology. The city of Sulaimaniyah in the Eastern Kurdish region of Northern Iraq has experienced an outbreak of 14 of these lesions over a period of 40 months. Isolated liver abscesses attributable to $F$. hepatica were found in 6 of the 14 patients with the parasite also being identified in local cattle and as an endemic infestation of snails in local streams. Ulcerating GI abscesses caused by a Zygomycete fungus consistent with basidiobolomycosis occurred in 6 of the 14 patients. These two diseases have not been previously reported as occurring at the same time in the same region. The mode of transmission of basidiobolomycosis to humans is unknown, but the emergence in Sulaimaniyah together with fascioliasis supports the suggestion that basidiobolomycosis may be transmitted by the feces of grazing animals [16].

\section{Limitations}

The study is observational and descriptive. As a descriptive study, it presents a relatively small number of patients that may bias generalizations regarding treatment, outcomes, and general manifestations of the diseases. The study is further limited by the lack of fungal cultures for the organism identified by histology as probable basidiobolomycosis.

\section{Abbreviations}

Gl: Gastrointestinal; ELISA: Enzyme linked immunoabsorbent assay; F. hepatica: Fascilola hepatica; PAS: Periodic acid Schiff.

\section{Competing interest}

The authors declare that they have no interests that compete with any of the contents of the manuscript.

\section{Authors' contributions}

$\mathrm{HAH}, \mathrm{RAM}, \mathrm{NGR}$, and BEN contributed to data collection and analysis. $\mathrm{HAH}$ and RAM wrote the first draft of the manuscript. RMR, KHK, and TAH contributed patient information and revision of the manuscript. ABF and JG consulted on the case material and identified the $\mathrm{Gl}$ disease as basidiobolomycosis. MDH, ABF, and JG helped write the several versions of the manuscript. The final version of the manuscript was edited and approved by all authors.

\section{Author details}

${ }^{1}$ Departments of Pathology, Medicine, and Surgery, Shorsh General Hospital, Sulaimaniyah, Iraq. ${ }^{2}$ Departments of Microbiology and Surgery, Sulaimaniyah University College of Medicine, Sulaimaniyah, Iraq. ${ }^{3}$ Ministry of Health, Kurdish Regional Government, Erbil, Iraq. ${ }^{4}$ Department of Pathology, Emory University, Atlanta, GA, USA.

Received: 25 September 2012 Accepted: 1 February 2013 Published: 20 February 2013

\section{References}

1. Rosai J (Ed): Rosai and Ackerman's Surgical Pathology. Ninth edition. Maryland Heights, Missouri: Mosby (Elsevier); 2004

2. Mills SE (Ed): Sternberg's Diagnostic Surgical Pathology. Fifth edition. Philadelphia: Wolters Kluwer (Lippincott Williams and Wilkins); 2010.

3. Silverberg SG, DeLellis RA, Frable WJ, LiVolsi VA, Wick MR: Silverberg's Principles and Practice of Surgical Pathology and Cytology. Fourth edition. Philadelphia: Churchill Livingston (Elseveir); 2006.

4. Odze RD, Goldblum JR (Eds): Surgical Pathology of the Gl tract, Liver, Biliary Tract, and Pancreas. Second edition. Philadelphia: Saunders (Elseveir); 2009. 
5. Fenoglio-Preiser CM, Noffsinger AE, Stemmermann GN, Lantz PE, Isaacson PG (Eds): Gastrointestinal Pathology: An Atlas and Text. Third edition. Philadelphia: Wolters Kluwer (Lippincott Williams and Wilkins); 2008.

6. Burt AD, Portman BC, Ferrell LD (Eds): MacSween's Pathology of the Liver. Fifth edition. Philadelphia: Churchill Livingston (Elsevier); 2007

7. Kim GB, Kwon JH, Kang DS: Hypereosinophilia syndrome: Imaging findings in patients with liver involvement. Amer J Radiol 1993, 161:577-580.

8. Lim JH, Lee KS: Eosinophilic infiltration in Korea: idiopathic? Korean J Radiol 2006, 7:4-6.

9. Jain R, Sawhney S, Bhargava DK, Panda SK, Berry M: Hepatic granulomas due to visceral larva migrans in adults: appearance on US and MRI. Abdom Imaging 1994, 19:253-256.

10. Rayes A, Teixeira D, Nobre V, Serufo JC, Gonçalves R, Valadares L, Lambertucci JR: Visceral larva migrans syndrome complicated by liver abscess. Scand J Infect Dis 1999, 31:324-329.

11. Hartleb M, Januszewski K: Severe hepatic involvement in visceral larva migrans. Eur J Gastroenterol Hepatol 2001, 13:1245-1249.

12. Musso C, Castelo JS, Tsanaclis AM, Pereira FE: Prevalence of Toxocarainduced liver granulomas, detected by immunohistochemistry, in a series of autopsies at a Children's Reference Hospital in Vitoria, ES, Brazil. Virchows Arch 2007, 450:411-417.

13. Kaplan KJ, Goodman ZD, Ishak KG: Eosinophilic granuloma of the liver: a characteristic lesion with relationship to visceral larva migrans. Amer J Sugical Pathol 2001, 25:1316-1321.

14. Vercelli-Retta J, Lagios MD, Chandrasoma P: Fasciola hepatica and parasitic eosinophilic granuloma of the Liver. Am J Surg Pathol 2002, 26:1238.

15. Acosta-Ferreira W, Vercelli-Retta J, Falconi LM: Fasciola hepatica human infection. Histopathological study of sixteen cases. Virchows Arch A Pathol Anat Histol 1979, 383:319-327.

16. Lyon GM, Smilack JD, Komatsu KK, Pasha TM, Leighton JA, Guarner J, Colby TV, Lindsley MD, Phelan M, Warnock DW, Hajjeh RA: Gastrointestinal basidiobolomycosis in Arizona: clinical and epidemiological characteristics and review of the literature. Clin Infect Dis 2001, 15:1448-1455.

17. Nemenqani D, Yaqoob N, Khoja H, Al Saif O, Amra NK, Amr SS: Gastrointestinal basidiobolomycosis: an unusual fungal infection mimicking colon cancer. Arch Pathol Lab Med 2009, 133:1938-1942.

18. Al Jarie A, Al-Mohsen I, Al Jumaah S, Al Hazmi M, Al Zamil F, Al Zahrani M, Al Modovar E, Al Dayel F, Al Arishii H, Shehrani D, Martins J, Al Mehaidib A Rossi L, Olaiyan I, Le Quesne G, Al-Mazrou A: Pediatric gastrointestinal basidiobolomycosis. Pediatr Infect Dis J 2003, 22:1007-1014

19. Vickram HR, Smilack JD, Crowell MD, De Petris G: Emergence of gastrointestinal basidiobolomycosis in the United States, with a review of worldwide cases. Clin Infect Dis 2012, 54:1685-1691.

20. Mas-Coma MS, Esteban JG, Bargues MD: Epidemiology of human fascioliasis: a review and proposed new classification. Bull World Health Organ 1999, 77:340-346.

21. Mas-Coma S, Valero MA, Bargues MD: Fasciola, lymnaeids and human fascioliasis, with a global overview on disease transmission, epidemiology, evolutionary genetics, molecular epidemiology and control. Adv Parasitol 2009, 69:141-146.

22. Mas-Coma S: Epidemiology of fascioliasis in human endemic areas. J Helminth 2005, 79:207-216.

23. Haseeb AN, El-Shazly AM, Arafa MA, Morsy AT: A review on fascioliasis in Egypt. J Egypt Soc Parasitol 2002, 32:317-354.

24. Parkinson M, O'Neil SM, Dalton JP: Endemic human fasciolosis in the Bolivian Altiplano. Epidem Infect 2007, 135:669-674.

25. Mas-Coma S: Epidemiology of fascioliasis in human endemic areas. J Helminthol 2005, 79:207-216.

26. Massoud J: Fascioliasis outbreak of man and drug tests (triclabendazole) in Caspian littoral, northern part of Iran. Bull Soc Fr Parasito 1989, I8:438.

27. Mailles A, Capek I, Ajana F, Schepens A, llef D, Vaillant D: Commercial watercress as an emerging source of fascioliasis in northern France in 2002. Epidemiol Infect 2006, 134:942-945.

28. Mas-Coma S, Bargues MD, Esteban JG: Human Fasciolosis, Chapter 12. In CAB International. Edited by Fasciolosis DJP. Cambridge: University Press; 1999:411-434

29. Geramizadeh B, Foroughi R, Keshtkar-Jahromi M, Malek-Hosseini SA, Alborzi A: Gastrointestinal basidiobolomycosis, an emerging infection in the immunocompetent host: a report of 14 patients. J Med Microbiol 2012, 61:1770-1774
30. Centers for Disease Control and Prevention (CDC): Gastrointestinal basidiobolomycosis-Arizona, 1994-1999. MMWR Morbid Mortal Wkly Rep 1999, 48:710-713.

31. AL-amran FGY: Surgical experience of 825 patients with thoracic hydatidosis in Iraq. Ind J Thoracic Cardiovasc Surg 2008, 24:124-128.

32. Sadjjadi SM: Present situation of echinococcus in the Middle East and Arabic North Africa. Parasitol Int 2006, 55:S197-S202.

33. Carnevale S, Rodríguez MI, Santillán G, Labbé JH, Cabrera MG, Bellegarde EJ, Velásquez JN, Trgovcic JE, Guarnera EA: Immunodiagnosis of human fascioliasis by an enzyme-linked immunosorbent assay (ELISA) and a micro-ELISA. Clin Diagn Lab Immunol 2001, 8:174-177.

34. Hillyer GV: Immunodiagnosis of human and animal fasciolosis, Chapter 13. In CAB International. Edited by Fasciolosis DJP. Cambridge: University Press; 1999:435-447.

35. Yazgan Aksoy D, Kerimoglu U, Erguven S, Arslan S, Unal S, Batman F Bayraktar Y: Fasciola hepatica infection: clinical and computerized tomographic findings of ten patients. Turk J Gastroenterol 2006, 17:40-45.

36. Ribes JA, Vanover-Sams CL, Baker DJ: Zygomycetes in Human Disease. Clinical Microbiol Rev 2000, 13:236-301.

37. National Institutes of Health (US): National Library of Medicine-Medical Subject Headings. MESH heading: Gastrointestinal tract; 2011. http://www.nIm. nih.gov/cgi/mesh/2013/MB_cg.

38. Hawramy TA, Saeed KA, Qaradaghy SH, Karboli TA, Nore BF, Bayati NH: Sporadic incidence of fascioliasis during hepatobiliary procedures: a study of 18 patients from Sulaimaniyah Governate. BMC Res Notes 2012, 5:691

39. Ashrafi K, Valero MA, Massoud J, Sobhani A, Solaymani-Mohammadi S, Conde $\mathrm{P}$, Khoubbane M, Baques MD, Mas-Coma S: Plant-borne human contamination by fascioliasis. Am J Trop Med Hyg 2006, 75:295-302.

40. Marcos LA, Tagle M, Terashima A, Bussalleu A, Ramirez C, Carrasco C, Valdez L, Huerta-Mercado J, Freedman DO, Vinetz JM, Gotuzzo E: Natural history, clinicoradiologic correlates, and response to triclabendazole in acute massive fascioliasis. Am J Trop Hyg 2008, 78:222-227.

41. Soliman MFM: Epidemiologic review of human and animal fascioliasis in Egypt. J Infect Developing Countries 2008, 2:182-189.

42. Fahimzad A, Karimi A, Tabatabaei SR, Zadeh MG: Gastrointestinal basidiobolomycosis as a rare etiology of bowel obstruction. Turk J Med Sci 2006, 36:239-241

43. van den Berk GEL, Noorduyn LA, van Ketel RJ, van Leeuwen J, Bemelman WA Prins JM: A fatal pseudo-tumor: disseminated basidiobolomycosis. BMC Infect Dis 2006, 6:140

44. El-Shabrawi MHF, Kamal NM: Gastrointestinal basidiobolomycosis in children: An overlooked emerging infection. J Med Microbiol 2011, 60:871-880.

45. Apt W, Aguilera X, Vega F, Miranda C, Zulantay I, Perez C, Gabor M, Apt P: Treatment of human fascioliasis with triclabendazol: drug efficacy and serological response. Am J Trp Med Hyg 1995, 52:532-535.

46. Magnaval J-F, Glickman LT, Dorchies P, Marassin B: Highlights of human toxocariasis. Korean J Parasitol 2001, 38:1-11

47. Watthanakulpanich D: Diagnostic trends of human toxocariasis. J Trop Med Parasitol 2010, 33:44-52.

48. Park Cl, Kim H, Ro JY, Gutierrez Y: Human ectopic fascioliasis in the cecum. Am J Surg Pathol 1984, 8:73-77.

49. Makay O, Gurcu B, Caliskan C, Nart D, Tuncyurek M, Korkut M: Ectopic fascioliasis mimicking a colon tumor. World J Gastroenterol 2007, 13:2633-2635.

50. Despommier D: Toxocariasis: Clinical aspects, epidemiology, medical ecology, and molecular aspects. Clinical Microbiol Rev 2003, 16:265-272.

doi:10.1186/1471-2334-13-91

Cite this article as: Hassan et al:: Eosinophilic granulomatous gastrointestinal and hepatic abscesses attributable to basidiobolomycosis and fasciolias: a simultaneous emergence in Iraq Kurdistan. BMC Infectious Diseases 2013 13:91. 